\title{
Long-term acclimation of barley photosynthetic apparatus to narrow-band red and blue light
}

\author{
G.V. KOCHETOVA ${ }^{+}$, O.B. BELYAEVA, D.S. GORSHKOVA, T.A. VLASOVA, E.M. BASSARSKAYA, \\ T.V. ZHIGALOVA, and O.V. AVERCHEVA
}

Faculty of Biology, M. V. Lomonosov Moscow State University, Moscow 119234, Russia

\begin{abstract}
Chloroplasts of barley plants grown under red light $(\mathrm{RL}, 660 \mathrm{~nm})$ dramatically differed from the chloroplasts of plants raised under blue light (BL, $450 \mathrm{~nm}$ ) or control plants (white light). The chloroplasts under RL had an extensive membrane system with high stacking degree and disordered irregular shaped stacks (shaggy-formed grana). After $5 \mathrm{~h}$ in darkness, dynamic rearrangements of chloroplast architecture in RL- and especially BL-grown plants were restricted compared with control plants. The light spectral quality affected the content and proportions of photosynthetic pigments. The leaves of RL-grown plants had the increased ratio of low-temperature fluorescence bands, $\mathrm{F}_{741} / \mathrm{F}_{683}$, corresponding to emission of PSI and PSII, respectively. This increase can be related to specific architecture of chloroplasts in RL-treated plants, providing close spacing between the two photosystems, which enhances energy transfer from PSII to PSI and facilitates the movement of LHCII toward PSI.
\end{abstract}

Additional key words: electron microscopy; LED; spectrofluorimetry; spillover.

\section{Introduction}

The structure of higher plant chloroplasts comprises a well developed system of internal membranes that produce thylakoids of two types: granal and stromal thylakoids. The thylakoid membranes contain transmembrane pigment-protein complexes of the two photosystems (PSI and PSII) together with the light-harvesting complexes, LHCII. LHCII antenna complexes, predominantly associating with PSII, absorb and transfer light energy to the reaction centers of the two photosystems. The two photosystems are laterally segregated. PSII is concentrated in stacked-membrane domains of the grana. PSI is found in stroma membranes (Albertsson 2001, Allen and Forsberg 2001, Danielsson et al. 2004, Kouřil et al. 2013, Ruban and Johnson 2015) and at the grana margins (the transition area where stromal thylakoids converge with the granal ones) (Mullineaux 2005). Such a segregation of membrane complexes enables their comparatively autonomous functioning along with regulated interactions.

The structural organization of photosynthetic membranes, their composition and location of functional complexes depends substantially on environmental conditions, primarily on light intensity and spectral quality
(Anderson et al. 1988, Eskins et al. 1989, Pribil et al. 2014). The light-induced changes are classified into fast (short-term) and slow (long-term) responses.

Fast responses to changes in light conditions consist in a dynamic rapid process associated with the lateral redistribution of LHCII within the thylakoid membrane; this process allows the plants to rapidly adjust the performance of the photosynthetic apparatus (PSA) to new light conditions. Being a short-term acclimation response, the process called state transition redistributes the amount of excitations between PSI and PSII. The distribution of the part of the mobile LHCII pool between the two photosystems depends on the redox state of electrontransport chain components, i.e. the plastoquinone pool and cytochrome $b_{6} / f$-complex, as well as on the redox state of stromal components (thioredoxin) (Tikkanen et al. 2006, 2008; Tikkanen and Aro 2012). When the excitation pressure for PSI is more than for PSII, almost all LHCII are associated with PSII (state 1) (Wientjes et al. 2013). When the excitation pressure changes in favor of PSII, a mobile pool of LHCII triggered by the protein phosphorylation moves and reversibly associates with PSI

Received 2 March 2017, accepted 9 May 2017, published as online-first 1 June 2017.

${ }^{+}$Corresponding author; e-mail: galina.kochetova@gmail.com

Abbreviations: BL - blue light; Car - carotenoids; Chl - chlorophyll; LEDs - light-emitting diodes; RL - red light; PSA - photosynthetic apparatus; WL - white light.

Acknowledgements: This work was supported by State Research Program (state registration No. AAAA-A16-116021660106-0 and No. AAAA-A16-116021660020-9) and by the Russian Foundation for Basic Research, project No. 14-04-31823mol_a: "Effects of narrow spectral bands of visible light on structural-functional conditions of energy-transducing systems in barley chloroplasts." 
(state 2) (Bonaventura and Myers 1969, Murata 1969, Allen 1992, Kouřil et al. 2005, Takahashi et al. 2006, Iwai et al. 2010, Papageorgiou and Govindjee 2011). As a result of this transition, a part of the mobile LHCII pool starts transferring energy to PSI, thus mitigating the imbalance of excitation pressure on two photosystems. The redistribution of LHCII is accompanied by a partial loosening of the grana stacks, which leads to thylakoid unstacking and the loss of regular granal structure (Chuartzman et al. 2008). At high light intensities the PSII complexes move toward unstacked thylakoids, which facilitates the repair of the damaged part of the PSII core complex (YoshiokaNishimura 2016). Thus, the dynamic rearrangements of the chloroplast ultrastructure promote the PSA adaptation to rapidly changing environmental conditions.

The long-term acclimation of plants proceeds under prolonged changes in light conditions. There are several mechanisms of long-term acclimation. When the imbalance in photoexcitation of the two photosystems persists for a longer period, plants adjust their PS stoichiometry (Chow et al. 1990, Melis 1991, Hogewoning et al. 2012). Upon the increase in light intensity, the acclimation reduces the content of major peripheral antenna proteins LHCII, which diminishes the amount of absorbed light quanta (Anderson and Andersson 1988, Tanaka and Tanaka 2000). In addition, the PSII-LHCII supercomplexes form comparatively loose semicrystalline arrays in the grana membrane, and thus reduce the

\section{Materials and methods}

Plants and growth conditions: The experiment was performed with 9-d-old seedlings of barley (Hordeum vulgare L., cv. Luch). The seeds were soaked in a diluted potassium permanganate solution for $3 \mathrm{~h}$ to initiate germination and remove possible pathogens. After this, they were placed into hydroponic vessels with Hoagland mineral solution $\left[5 \mathrm{mM} \mathrm{Ca}\left(\mathrm{NO}_{3}\right)_{2}, 1 \mathrm{mM} \mathrm{KH} \mathrm{KO}_{4}, 1 \mathrm{mM}\right.$ $\mathrm{MgSO}_{4}, 5 \mathrm{mM} \mathrm{KNO}{ }_{3}, 0.09 \mathrm{mM}$ Fe-EDTA] supplemented with $0.78 \mathrm{mM}$ Mes-NaOH buffer ( $\mathrm{pH}$ 6.5) and transferred to growth chambers for their respective light treatments. Plants were grown with two types of LEDs: red (emission maximum at $660 \mathrm{~nm}$, half-width $16 \mathrm{~nm}$; RL) or blue (450 nm, half-width $24 \mathrm{~nm}$; BL). Plants grown under white fluorescent lamps (WL) were used as a control group. The photosynthetic photon flux at the top of the leaves was approximately $70 \mu \mathrm{mol} \mathrm{m} \mathrm{m}^{-2} \mathrm{~s}^{-1}$, the photoperiod was $16-\mathrm{h}$ light/8-h dark. Emission spectra of the light sources used (Fig. 1) were obtained with an AvaSpec-2048 spectrometer using AvaSoft (C) 7.8.0 software (Avantes BV, the Netherlands). Ambient temperature in the growth chamber was $22-23^{\circ} \mathrm{C}$.

The leaf segments of $2 \mathrm{~cm}$ in length were excised from the first true leaves at a distance of $2 \mathrm{~cm}$ from the apex. The leaves were sampled immediately under growth light conditions $(6 \mathrm{~h}$ after dawn, the treatment designated $0 \mathrm{hD}$, efficiency of energy transfer (Dekker and Boekema 2005, Kouřil et al. 2013).

Recently it was shown that at natural light conditions LHCII serves as an antenna of both PSI and PSII (Galka et al. 2012, Wientjes et al. 2013). This allows an acclimation to different light intensities to be performed simply by regulating the expression of LHCII genes only.

The ultrastructure of thylakoid membranes is formed in accordance with the intensity and spectral composition of light. In plants grown under low light intensity or red light, the chloroplasts exhibit a high thylakoid frequency per chloroplast section and high stacking degree, which is due to the hydrophobicity of the peripheral pigment-protein LHCII complexes. In plants grown under high-intensity light or blue light, the chloroplasts possess low amounts of thylakoids per chloroplast section and a low stacking degree (Vlasova and Osipova 1973, Deng et al. 1989, Lichtenthaler and Burkart 1999, Liu et al. 2011, Su et al. 2014).

In this work, we studied the action of LED-emitted narrow-band red and blue light on PSA organization in barley seedlings. In order to clarify the mechanisms of long-term acclimation of plants to monochromatic illumination, we examined the chloroplast ultrastructure by electron microscopy, the state of pigment-protein complexes by means of low temperature $(77 \mathrm{~K})$ chlorophyll fluorescence, and assessed the dynamics of these characteristics during dark adaptation.

"in the light"). In addition, the leaves were sampled from plants adapted to darkness for 1,3 , and $5 \mathrm{~h}$ (the treatments designated as $1 \mathrm{hD}, 3 \mathrm{hD}$, and $5 \mathrm{hD}$, respectively, "in the dark").

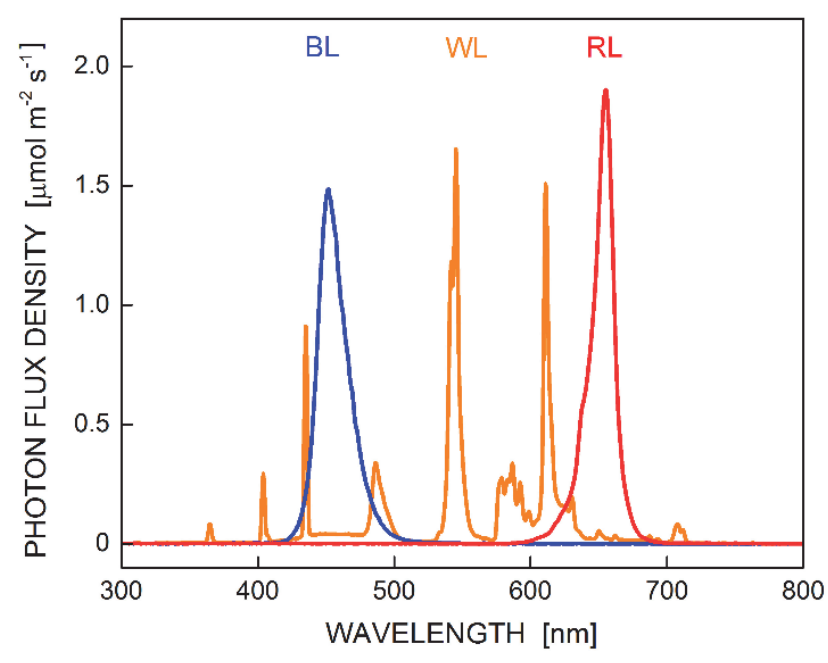

Fig. 1 Emission spectra of the irradiance sources used in this study [normalized to $70 \mu \mathrm{mol}$ (photon) $\mathrm{m}^{-2} \mathrm{~s}^{-1}$ by the integral area]. RL: $\lambda_{\max }=660 \mathrm{~nm}, \Delta \lambda^{1 / 2}=16 \mathrm{~nm}$; BL: $\lambda_{\max }=450 \mathrm{~nm}$, $\Delta \lambda \frac{1}{2}=24 \mathrm{~nm}$; WL: white fluorescent lamp. 
Electron microscopy: The leaf cuts of $1 \times 1 \mathrm{~mm}$ were sampled aside from the midrib and used for electron microscopy studies. The material was fixed with $2.5 \%$ glutaraldehyde solution (Reanal, Hungary) in phosphate buffer $(\mathrm{pH} 7.4)$, postfixed with $1 \%$ solution of $\mathrm{OsO}_{4}$ (Serva, Germany) in the same buffer, dehydrated in a series of ethanol solutions, with ethanol concentrations increasing from 30 to $100 \%$. The $70 \%$ ethanol solution was supplemented with $1 \%$ uranyl acetate, and the samples were left overnight in this solution. After dehydration, the samples were transferred to absolute acetone and embedded into epoxy resin mixture containing Epon 812, dodecenyl succinic anhydride (DDSA), methyl nadic anhydride (MNA), and tris(dimethylaminomethyl)phenol (DY064) catalyst at a volume ratio of 13:8:7:1 (Fluka AG, Switzerland) (Weakley 1972). The sections were cut with a diamond knife using an LKB-4800 Ultratome $V$ ( $L K B$, Sweden), mounted on copper mesh grids, and contrasted with lead citrate according to Reynolds (Reynolds 1963). The samples were examined under a JEM-1011 transmission electron microscope (JEM-1011 and JEM-100B, $J E O L$, Japan) at the Laboratory of Electron Microscopy, Faculty of Biology, Moscow State University.

Pigment content: The pigment content in leaves was determined using 100\% acetone extracts and a Specord 200 spectrophotometer (Analytik Jena, Germany). The pigments content was quantified according to Lichtenthaler (1987). The leaf pigment content was expressed per unit of leaf area.

Low-temperature (77K) fluorescence spectroscopy: Spectral studies were carried out on the material of leaf homogenates to avoid the effects of light reabsorption. In order to prepare the homogenates, leaves were ground with a buffer (0.025 M Tris-HCl, $\mathrm{pH} 8.0 ; 0.35 \mathrm{M} \mathrm{NaCl})$ on ice. After the grinding the homogenates were filtered through

\section{Results}

Chloroplast ultrastructure: Analysis of chloroplast ultrastructure by transmission electron microscopy (Fig. 2) showed that chloroplasts of illuminated control plants possessed a granular stroma and clearly distinguished grana, from 12 to 30 grana stacks per chloroplast section. The grana sections had a rectangular shape and consisted on average of 9 to 15 thylakoids (up to 30 thylakoids per granum). The incubation of control plants in darkness for $5 \mathrm{~h}$ produced significant changes in the organization of chloroplast membrane structure: the proportion of stromal thylakoids increased at the expense of reduced free space of stroma; the lumenal gap expanded significantly, while the granum sections retained their rectangular shape.

In plants grown under BL, the membrane structure of illuminated chloroplasts was similar to that in the control WL treatment. The cross-sections of grana had a rectan- a nylon cloth with the mesh size of $\sim 50 \mu \mathrm{m}$, and glycerol was added to a final concentration of $65 \%(\mathrm{v} / \mathrm{v})$. Portions of these homogenates were gently frozen in liquid nitrogen to obtain a transparent sample and then used for measurement. The chlorophyll (Chl) content of the samples estimated by the method of Arnon (1949) was 8-10 $\mu \mathrm{g}$ $\mathrm{mL}^{-1}$, and the sample width was $1.5 \mathrm{~mm}$.

Low-temperature $(77 \mathrm{~K})$ fluorescence emission and excitation spectra of leaf homogenates were measured with a FluoroMax-4 spectrofluorometer (HORIBAScientific, France). For emission spectra, the fluorescence was excited at $440 \mathrm{~nm}$. The width of the entrance and exit slits was $2 \mathrm{~nm}$. After subtracting the background signal, the spectra were normalized to the maximum at $683 \mathrm{~nm}$.

The excitation spectra of light-harvesting complexes of PSI were recorded by measuring fluorescence emission at $735 \mathrm{~nm}$. The spectra (Figs. 5,6) were normalized at $705 \mathrm{~nm}$, the PSI-LHCI terminal emitter region, where there is no absorption by PSII or LHCII and where PSI fluorescence is still missing (Benson et al. 2015). Excitation spectra of the LHC of PSII were recorded for emission at $693 \mathrm{~nm}$ and normalized at $533 \mathrm{~nm}$. The width of the entrance and exit slits was $1 \mathrm{~nm}$.

Statistical analysis: The pigment composition was determined in four independent experiments made in duplicate per treatment $(n=8)$, each with three leaves sampled from different plants. The $P<0.05$ was considered as statistically significant. The low temperature fluorescence was determined with three leaves sampled from different plants: $n=6-8$ independent experiments for emission spectra $1 \mathrm{hD}, 3 \mathrm{hD}, 5 \mathrm{hD}, n=12-14$ experiments for emission spectra $0 \mathrm{hD}$, and $n=5-6$ independent experiments for excitation spectra per treatment. Figures were completed using OriginPro 2015. Significant differences of numerical data represented in tables were estimated using the Mann-Whitney's $U$-test.

gular shape with clear borders; the grana on average contained 10-16 thylakoids (up to 40 in some cases), while the number of stromal thylakoids was comparatively low. After a 5-h dark period, the structural organization of the internal chloroplast membranes remained largely unchanged.

In plants grown under RL, the architecture of illuminated chloroplasts had an irregular structure. As compared to the chloroplasts of control plants, the chloroplasts of RL-grown plants featured a distinctly increased number of thylakoid membranes and the extended length of stacked regions in the absence of clear thylakoid organization into grana stacks (shaggy-formed grana). Grana were larger in diameter and had an irregular shape with numerous protruding thylakoids; the fusions of neighboring grana were frequently noted. The thylakoid arrangement was disordered in some regions. The normal parallel alignment 

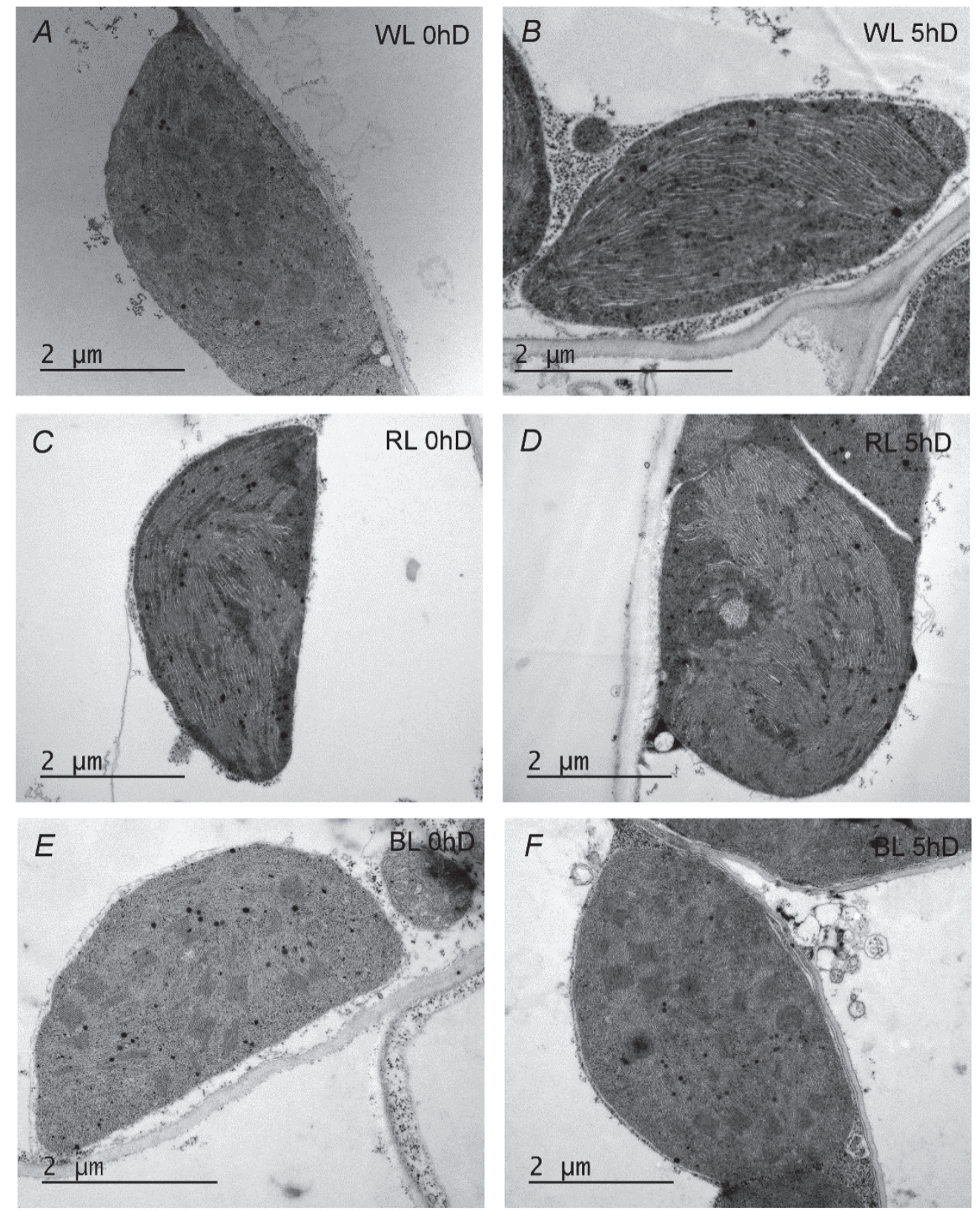

Fig. 2 Electron micrographs of chloroplasts from 9-d-old barley leaves grown under: $A, B$ - white light of fluorescent lamps (WL - control), $C, D$ - red light, $660 \mathrm{~nm}(\mathrm{RL}), E, F$ - blue light, $450 \mathrm{~nm}(\mathrm{BL}) . A, C, E$ - samples fixed directly under growth light conditions $(0 \mathrm{hD}) ; B, D, F$ - samples fixed after $5 \mathrm{~h}$ in darkness $(5 \mathrm{hD})$.

of the membrane system was disturbed: there were curved thylakoids, and the grana were oriented at different angles. In some cases the lumenal gap significantly increased. On average, the grana comprised 12-20 (up to 30) thylakoids. In darkness, the organization of the chloroplast membrane structure remained mostly unchanged, but the disordered positioning of the internal membranes increased slightly.

Pigment content: Determinations of $\mathrm{Chl} a, b$, and carote noid (Car) content revealed the largest amounts of $\mathrm{Chl} a$, Chl $b$, and Car in leaves of WL-grown plants (Table 1). The pigment content in leaves of RL-grown plants was $10-13 \%$ lower, while the lowest pigment content was observed in the leaves of BL-grown plants (14-20\% lower than in control plants). The $\mathrm{Chl} / \mathrm{Car}$ ratios in all the treatments were nearly equal, while the $\mathrm{Chl} a / b$ ratio was minimal in WL-grown plants and was maximal in BLgrown plants. 
Table 1. The content and ratios of photosynthetic pigments in the first leaf of 9-d-old barley seedlings. Designations WL 0hD, RL 0hD, and $\mathrm{BL} 0 \mathrm{hD}$ signify that the samples were taken directly under growth light conditions (white light of fluorescent lamps and emission of red and blue LEDs, respectively). Designations WL 5hD, RL 5hD, and BL 5hD apply to the same plants after dark adaptation for $5 \mathrm{~h}$. Data represent mean values of four experiments made in duplicate \pm SE. The same small letters in each parameter indicate insignificant differences at $P<0.05$ for plants without dark adaptation. The same capital letters in each parameter indicate insignificant differences at $P<0.05$ for plants with dark adaptation. $\mathrm{Chl}$ - chlorophyll, $\mathrm{Car}$ - carotenoids.

\begin{tabular}{lllllll}
\hline $\begin{array}{l}\text { Growth light } \\
\text { treatment }\end{array}$ & \multicolumn{2}{l}{ Pigment content $\left[\mathrm{mg} \mathrm{dm}^{-2}\right]$} \\
\hline Chl $a$ & Chl $b$ & Chl $(a+b)$ & Car & Chl $a / b$ & Chl $(a+b) / C a r$ \\
\hline WL 0hD & $3.35 \pm 0.14^{\mathrm{a}}$ & $1.08 \pm 0.06^{\mathrm{a}}$ & $4.44 \pm 0.20^{\mathrm{a}}$ & $0.81 \pm 0.03^{\mathrm{a}}$ & $3.07 \pm 0.04^{\mathrm{a}}$ & $5.51 \pm 0.06^{\mathrm{a}}$ \\
WL 5hD & $2.87 \pm 0.09^{\mathrm{A}}$ & $0.97 \pm 0.02^{\mathrm{A}}$ & $3.84 \pm 0.12^{\mathrm{A}}$ & $0.70 \pm 0.02^{\mathrm{A}}$ & $2.91 \pm 0.02^{\mathrm{A}}$ & $5.49 \pm 0.02^{\mathrm{A}}$ \\
RL 0hD & $3.01 \pm 0.02^{\mathrm{b}}$ & $0.93 \pm 0.02^{\mathrm{b}}$ & $3.94 \pm 0.03^{\mathrm{b}}$ & $0.72 \pm 0.01^{\mathrm{b}}$ & $3.20 \pm 0.06^{\mathrm{ab}}$ & $5.48 \pm 0.05^{\mathrm{a}}$ \\
RL 5hD & $2.80 \pm 0.04^{\mathrm{A}}$ & $0.89 \pm 0.02^{\mathrm{AB}}$ & $3.69 \pm 0.05^{\mathrm{A}}$ & $0.67 \pm 0.01^{\mathrm{AB}}$ & $3.09 \pm 0.04^{\mathrm{B}}$ & $5.54 \pm 0.03^{\mathrm{A}}$ \\
BL 0hD & $2.80 \pm 0.01^{\mathrm{c}}$ & $0.86 \pm 0.01^{\mathrm{c}}$ & $3.66 \pm 0.01^{\mathrm{c}}$ & $0.69 \pm 0.01^{\mathrm{c}}$ & $3.27 \pm 0.06^{\mathrm{b}}$ & $5.29 \pm 0.04^{\mathrm{b}}$ \\
BL 5hD & $2.66 \pm 0.04^{\mathrm{A}}$ & $0.84 \pm 0.01^{\mathrm{B}}$ & $3.50 \pm 0.03^{\mathrm{B}}$ & $0.65 \pm 0.01^{\mathrm{B}}$ & $3.09 \pm 0.02^{\mathrm{B}}$ & $5.39 \pm 0.03^{\mathrm{B}}$ \\
\hline
\end{tabular}

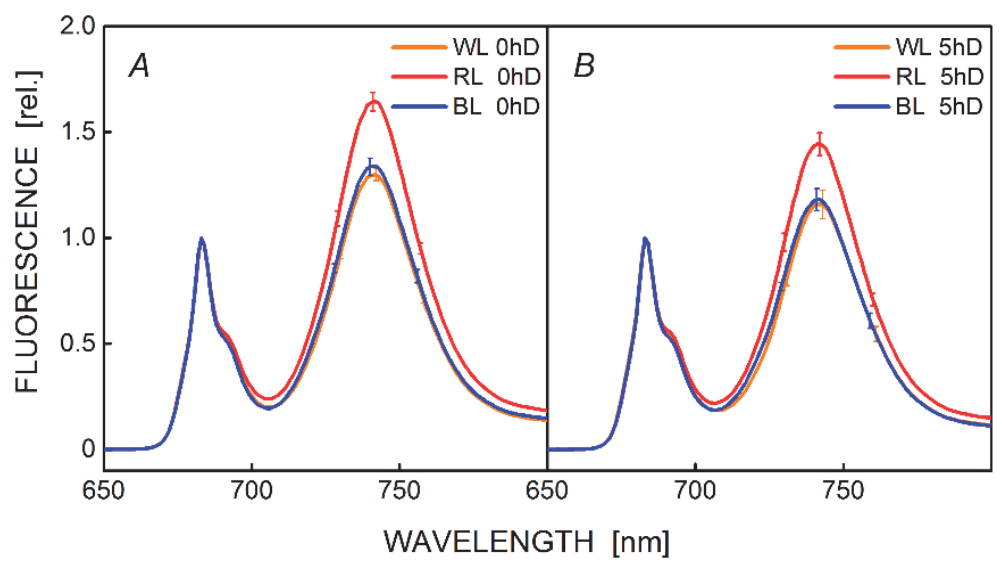

Fig. 3. Spectra of low-temperature fluorescence emission in the homogenates of barley leaves maturing at different light conditions: WL - white light, $\mathrm{RL}$ - red light, $660 \mathrm{~nm}, \mathrm{BL}$ - blue light, $450 \mathrm{~nm}$. $(A)$ 0 h dark, $(B)$ 5-h dark. Excitation at $440 \mathrm{~nm}$. Error bars represent SE.

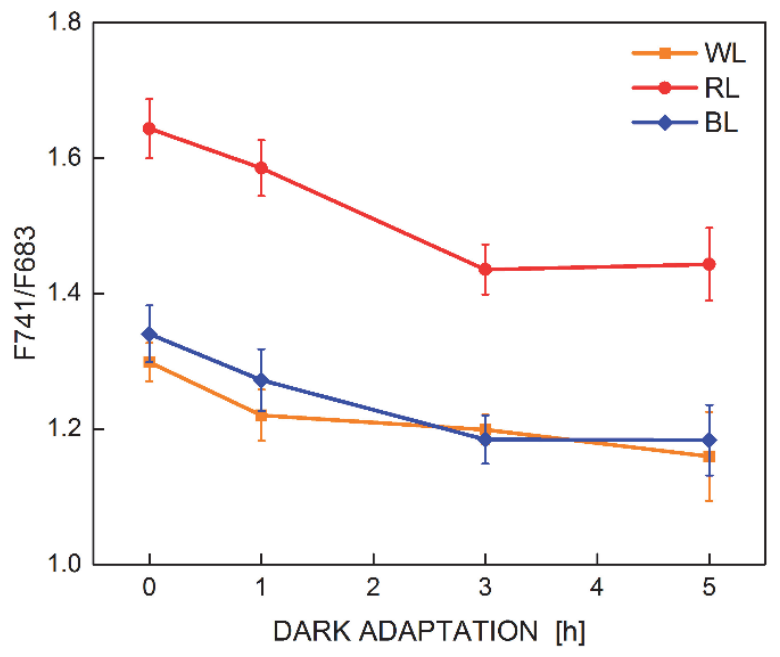

Fig. 4. Changes in $\mathrm{F}_{741} / \mathrm{F}_{683}$ ratio of low-temperature fluorescence during dark adaptation of barley seedlings grown under different light conditions: $\mathrm{WL}$ - white light; RL - red light, $660 \mathrm{~nm}$; BL blue light, $450 \mathrm{~nm}$. Excitation at $440 \mathrm{~nm}$.

The leaf pigment content was determined in all the treatments after 5-h plant adaptation to darkness. The quantitative content of pigments responded to transfer of plants to darkness (Bennett 1981, Chekunova and Savelieva 2010) and was also subject to daily variations
(Beator et al. 1992). In order to eliminate the influence of circadian rhythms, simultaneous assays of pigment content were performed in plants remaining exposed to growth light conditions. During this light exposure, the plant pigment content remained practically unchanged (Table $1 \mathrm{~S}$, supplement available online), which excluded the influence of circadian changes in the analyzed period. After 5-h period of darkness, the amounts of Chl $a, b$, and Car declined in all the plants tested (Table 1). The largest reduction (on average by $13 \%$ ) was observed in leaves of WL-grown plants; in RL- and BL-grown plants, the leaf pigment content was reduced by 6 and $4.5 \%$, respectively. Because of these changes, the differences in pigment content between the treatments were levelled after 5-h darkness. Nevertheless, the largest content of Chl $a, b$, and Car was found in leaves of WL-grown plants, and the smallest content was observed in BL-grown plants.

Interestingly, the content of Chl $a$ and Car was reduced in darkness to equal extents for plants in all the treatments (by 14, 7, and 5\% for WL-, RL-, and BL-grown plants, respectively). The content of $\mathrm{Chl} b$ decreased to lesser extents (by 10, 4, and 2\% in WL-, RL-, and BL-grown plants, respectively). Consequently, the $\mathrm{Chl} a / b$ ratio displayed a similar decrease upon darkening in all the treatments (by 5, 3, and 5\% in WL-, RL-, and BL-grown plants, respectively), whereas the $\mathrm{Chl} / \mathrm{Car}$ ratio was hardly changed. 


\section{Low-temperature (77K) fluorescence spectra}

Emission spectra: Low-temperature fluorescence spectra for homogenates of green leaves are characterized by three main peaks positioned near 685,693 , and $735-740 \mathrm{~nm}$. The short-wavelength peaks (685 and $693 \mathrm{~nm}$ ) belong to LHC of PSII, and the peak at $735 \mathrm{~nm}$ is due to the LHC of PSI (Krause and Weis 1991). The changes in the $\mathrm{F}_{735} / \mathrm{F}_{685}$ ratio estimated from low-temperature $\mathrm{Chl}$ fluorescence spectra reflect the changes in the excitation energy distribution between both photosystems (Murata 1969, Butler 1977, Benson et al. 2015). In our experiments with barley seedlings, the main peaks of chloroplast lowtemperature fluorescence were located at 683, 693, and $741 \mathrm{~nm}$. The $\mathrm{F}_{741} / \mathrm{F}_{683}$ ratio in the emission spectra of homogenates showed no significant difference between BL- and WL treatments, whereas RL samples showed a large increase $(\sim 25 \%)$ in this ratio compared to $\mathrm{WL}$ and BL samples (Fig. 3A). This difference was observed for plants taken directly from the light chamber $(0 \mathrm{hD})$ and for the dark-adapted $(5 \mathrm{hD})$ plants (Fig. $3 A, B)$. For all growth light treatments the fluorescence ratio decreased after darkening by $11-12 \%$ as compared to the initial readings obtained immediately after illumination (0hD) (Fig. 4). However, the $\mathrm{F}_{741} / \mathrm{F}_{683}$ ratio was still $20-30 \%$ higher for RL-grown plants than that for BL- and WL-grown plants.

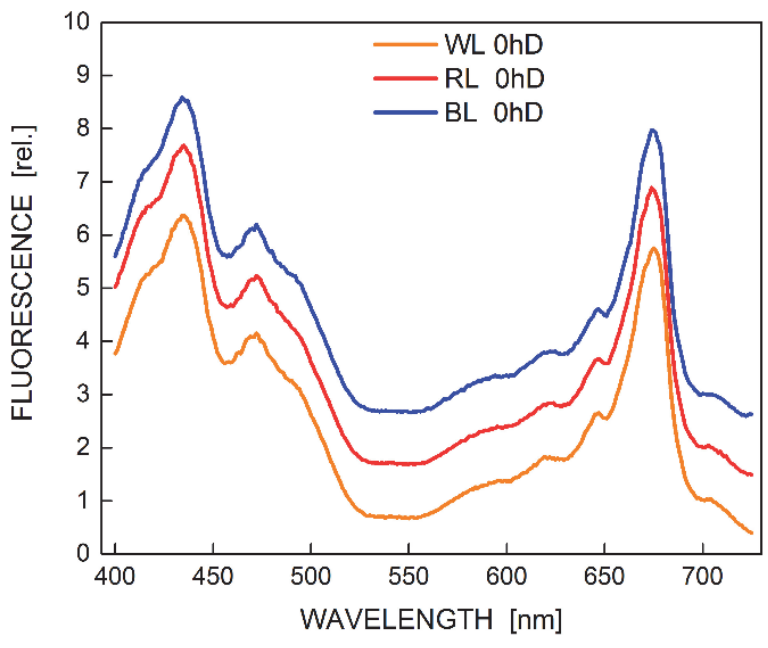

Fig. 5. Excitation spectra of PSI low-temperature fluorescence (emission at $735 \mathrm{~nm}$ ) in homogenates from barley leaves maturing at different light conditions $(0 \mathrm{hD}-$ without dark adaptation): $\mathrm{WL}$ - white light; RL - red light, $660 \mathrm{~nm} ; \mathrm{BL}$ - blue light, $450 \mathrm{~nm}$. All three graphs are almost identical. In order to avoid their overlap they were shifted along the $\mathrm{Y}$ axis.

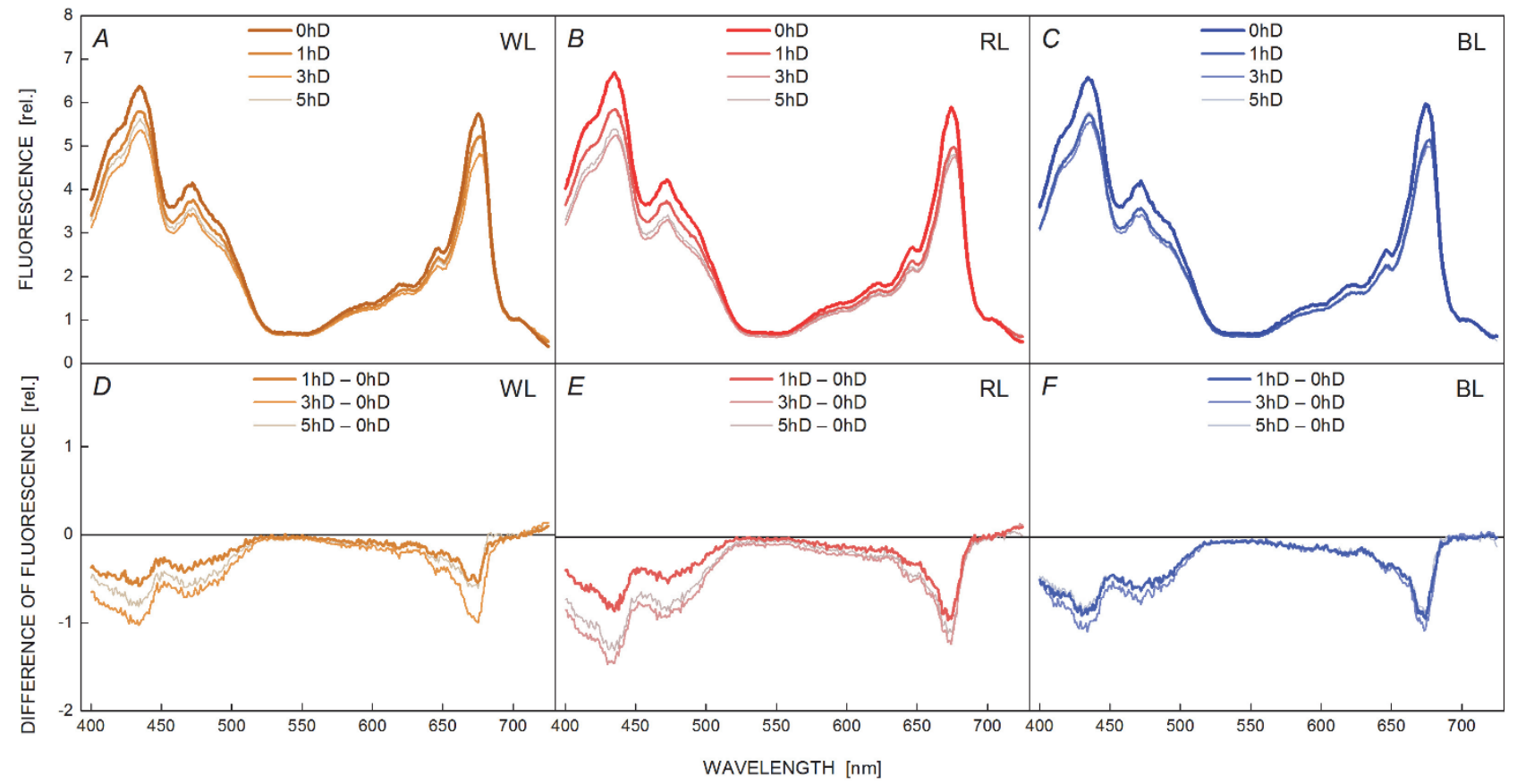

Fig. 6. Changes in low-temperature fluorescence excitation spectra of PSI (emission at $735 \mathrm{~nm}$ ) after darkening of plants grown at different light conditions: $(A, B, C)$ fluorescence excitation spectra (normalized spectra at $705 \mathrm{~nm}$ were recorded in $0,1,3$, and $5 \mathrm{~h}$ after darkening); $(D, E, F)$ subtracted spectra $(1 \mathrm{hD}$ minus $0 \mathrm{hD} ; 3 \mathrm{hD}$ minus $0 \mathrm{hD} ; 5 \mathrm{hD}$ minus $0 \mathrm{hD}$; see Table 1 for designations); $(A, D) \mathrm{WL}-$ white light of fluorescent lamps; $(B, E) \mathrm{RL}$ - red light, $660 \mathrm{~nm} ;(C, F) \mathrm{BL}$ - blue light, $450 \mathrm{~nm}$.

Excitation spectra were almost identical for all three light treatments applied during plant growth (Fig. 5). The transfer of plants to darkness induced similar modifications in the excitation spectra of PSI fluorescence for all three growth light treatments (WL, RL, and BL). A discernible decrease in the amplitude of the blue and red bands was noted, and the red band shifted by $2 \mathrm{~nm}$ from 674-675 nm to 676-677 nm (Fig. 6, Table 2S, supplement 
available online). Such changes in excitation (absorption) spectra indicate that the mobile antenna of LHCII retreated from PSI (Wientjes et al. 2013, Benson et al. 2015). As compared to the excitation spectrum of PSI, the excitation

\section{Discussion}

Chloroplast ultrastructure: Examination of the chloroplast ultrastructure by means of transmission electron microscopy revealed that chloroplasts of illuminated barley plants grown under fluorescent lamps exhibited a well-known differentiation into grana and stroma thylakoids (Fig. 2), which is consistent with the results obtained by other researchers (Pfeiffer and Krupinska 2005, Legocka et al. 2015). The chloroplasts developed under the light provided with blue LEDs had a highly organized structure, similar to that in control plants. Similar results were obtained for leaves of cucumber $(\mathrm{Su}$ et al. 2014) and tomato (Liu et al. 2011). The chloroplast membrane structure in plants grown under red light differed dramatically from that in WL-grown and BLgrown plants. The chloroplasts of the RL-grown plants were somewhat similar to chloroplasts of plants grown under low-intensity white light (Lichtenthaler and Burkart 1999): they featured broad grana stacks, very high grana stacks, and high stacking degree. However, the disordered orientation of chloroplast thylakoids was specific for RLgrown plants; it was absent in plants grown at lowirradiance white light, but was generally consistent with the data obtained for cucumber plants ( $\mathrm{Su}$ et al. 2014). Significant differences in the chloroplast structure were also observed under light conditions optimized for absorption of PSI or PSII (Melis 1984, Wagner et al. 2008) and in the mutant plants with impaired LHCII and photosystem complexes (Ladygin and Semenova 2005, Belgio et al. 2015).

During the 5-h dark period, the chloroplast structure formed under narrow-band blue light remained essentially unchanged, and the structure developed under red light underwent only slight changes. At the same time, the structure of chloroplasts in WL-grown control plants was subject to significant changes. The stromal thylakoids become more numerous, thus diminishing the free space of stroma, and the lumenal gap increased markedly. The chloroplast structural changes in WL-grown barley plants during the dark period were also observed by other researchers (Pfeiffer and Krupinska 2005). They noted that the chloroplast thylakoids of WL-grown plants were loosely stacked in the dark period and seemed to be swollen.

Thus, the system of internal membranes in chloroplasts developed under narrow-band red and blue light was less dynamic than in the control plants. The ability of plant chloroplasts to rearrange their membrane structure was reportedly lower in plants grown under high-intensity light than in plants grown under low irradiance (Vlasova and Osipova 1973). spectrum of PSII fluorescence (emission at $693 \mathrm{~nm}$ ) underwent much smaller changes during dark adaptation of plants; no shift of spectral lines was noticed (Fig. 1S, supplement available online).

Pigment content: Our assays of pigment content in the first leaf (Table 1) revealed that the WL-grown plants were characterized by the highest amount of pigments and by the lowest Chl $a / b$ ratio. The BL- and RL-grown plants. displayed similar $\mathrm{Chl} a / b$ ratios, but the Chl content in RLgrown plants was $7 \%$ higher than that in BL-grown plants.

Similar patterns of Chl content as a function of light quality used during plant growth were obtained with cucumber leaves ( $\mathrm{Su}$ et al. 2014). Some researchers detected the opposite pattern of $\mathrm{Chl}$ content: the maximum content was found in plants grown in blue light; intermediate, in red light; and the minimum in white light (Leong and Anderson 1984, Liu et al. 2012). However, these works were performed with other plant species grown at somewhat different light intensities and wavelengths.

In the case of insignificant differences between $\mathrm{Chl} a / b$ ratios, the total $\mathrm{Chl}$ content represents a more meaningful indicator than this ratio (Laisk et al. 2014). Based on these data, we assume a somewhat higher amount of LHCII in WL-grown plants, as compared to the RL- and, especially, BL-grown plants. Since photosynthetic pigments absorb red and blue quanta most effectively, the plants may sense such monochromatic light as more intense, as compared to white light of the same photon flux density. This could lead to the reduction of LHCII pool in plants grown under monochromatic light. The increase in light intensity was found to reduce the total pigment content and elevate the Chl $a / b$ ratio (Lichtenthaler and Burkart 1999).

However, the data on pigment content and ratios (Table 1) may also indicate the different amounts and proportions between the reaction centers of PSI and PSII and the respective LHCs (Laisk et al. 2014) for the treatments analyzed in our study.

The content of pigments decreased after 5-h darkness in all plant groups. The maximum reduction was observed in the control (WL) plants (13\%), which is consistent with the most pronounced changes in chloroplast ultrastructure of WL-grown plants after their transfer to darkness. In BLand RL-grown plants, the pigment content reduced by 4.5 and $6 \%$, respectively; the smallest changes occurred in BLplants. This is in line with the lack of ultrastructural changes upon darkening in chloroplasts of BL-plants and with the occurrence of minimal changes in RL-plants (see electron micrographs). Studies performed with various plant materials have shown that the transfer of plants from light to darkness led to reduction in Chl content (Bennett 1981, Chekunova and Savelieva 2010).

Thus, growing barley plants under blue light conferred the highest stability to pigments of LHCs and ensured the 
retention of thylakoid structural organization. A somewhat lower stability of these parameters was noted in RL-grown plants. The most labile pigment composition and the chloroplast membrane system were found in WL-grown control plants.

Low-temperature (77K) Chl fluorescence: The observed influence of growth light quality on low-temperature fluorescence spectra in barley leaf homogenates is a manifestation of long-term acclimation of plants to light conditions. Our studies showed that the plants grown under red light, as compared to $\mathrm{WL}$ and $\mathrm{BL}$, experienced the largest changes in fluorescent parameters and the chloroplast structure.

Judging by the action spectra for quantum yields of PSI and PSII (Laisk et al. 2014), both photosystems operate with equal efficiency in the blue light $(450 \mathrm{~nm})$, while the efficiency of PSII is $35 \%$ higher than that of PSI in the red light $(660 \mathrm{~nm})$. The resulting imbalance in electrontransport chain operation is leveled by significant adaptations occurring in the photosynthetic apparatus. The following long-term acclimation mechanisms are distinguished: (1) redistribution of LHCII mobile pool between the photosystems (Wientjes et al. 2013); (2) readjustment of LHCII amounts (Anderson and Andersson 1988, Tanaka and Tanaka 2000, Kouřil et al. 2013); (3) changes in stoichiometry of the two photosystems (Chow et al. 1990, Melis 1991, Hogewoning et al. 2012); (4) changes in characteristic densities and lattice parameters of semicrystalline arrays of PSII supercomplexes in grana membranes, affecting the efficiency of excitation energy transfer from the antennas to the reaction centers and between neighboring PSII complexes (Dietzel et al. 2011, Kouřil et al. 2013); and (5) changes in energy transfer from PSII to PSI by the mechanism of spillover associated with alterations in the chloroplast membrane structure (Mullineaux 2005).

The $\mathrm{F}_{741} / \mathrm{F}_{683}$ ratio characterizes the energy interaction of PSII and PSI. The discovered variations of this ratio for different light conditions (Fig. 3) may help to clarify the mechanism of photosynthetic adaptation in barley plants to narrow-band irradiance. In plants grown under red light, the $\mathrm{F}_{741} / \mathrm{F}_{683}$ ratio was $\sim 25 \%$ higher than that in BL- and WL-grown plants, both in the light $(0 \mathrm{hD})$ and after dark adaptation $(1,3$, and $5 \mathrm{hD})$. Specific features of chloroplast ultrastructure revealed in RL-grown plants were probably favorable for close spacing of the two photosystems. Small separation distances might promote energy transfer from PSII to PSI (spillover) and facilitate the movement of mobile LHCII pool to PSI, thus ensuring the largest $\mathrm{F}_{741} / \mathrm{F}_{683}$ ratio observed in this (RL) treatment. Although the fluorescence spectra in BL- and WL- treatments were identical, the fraction of PSI associated with LHCII may differ, because the $\mathrm{F}_{741} / \mathrm{F}_{683}$ ratio does not always match this fraction in quantitative terms (Wientjes et al. 2013).

The excitation spectra of PSI fluorescence were almost identical for all three light treatments (Fig. 5). The changes observed after dark adaptation were also identical: the amplitudes of spectral bands declined in all the treatments, and the red peak of PSI fluorescence excitation shifted to longer wavelengths (Fig. 6). Such changes in fluorescence excitation spectrum, as well as the decrease in $\mathrm{F}_{741} / \mathrm{F}_{683}$, ratio indicate the detachment of mobile LHCII from PSI (Hogewoning et al. 2012, Wientjes et al. 2013, Benson et al. 2015, Ware et al. 2015) and, consequently, the lowering of energy transfer to PSI. Hence, a part of the mobile LHCII in the light is associated with PSI, irrespective of the light treatments applied. Wientjes et al. (2013) showed that at a wide range of light intensities some part of the mobile LHCII is associated with PSI and serves as an antenna of both PSI and PSII. Thus, plants can adapt to both light quality and quantity by means of associating of a part of the mobile LHCII trimers with PSI.

The LHCII complexes detached from PSI either get connected to PSII, thus increasing the PSII antenna size, or remain unbound to any photosystems and produce aggregates, or may be destined to destruction. The dark destruction of pigment-protein complexes is evidenced by our findings that the pigment content was reduced in darkness in all the treatments (Table 1). However, the dark decay of the pigment content in RL- and, especially, BLgrown plants was significantly lesser than that in the control (WL-grown) plants. Thus, the dark degradation of pigment-protein complexes and the dark-induced reorganization of thylakoid membranes, revealed by electron microscopy (Fig. 2), proceeded differently in plants grown under different light conditions.

Conclusion: Our study demonstrated different properties of PSA developed during plant growth under white, blue, and red light. Judging by the pigment content and the reduced lability (increased stability) of the membrane structure, the BL-grown plants were similar to plants grown under RL. Considering the chloroplast ultrastructure and spectral characteristics, the BL-grown plants were similar to the control plant group (WL-grown plants). Thus, the use of red light during plant growth led to the formation of PSA having some similarities with that of BL-grown plants, but sharply different from the WLgrown plants. Remarkably, the BL-grown plants were less different from the WL-plants. Judging from lowtemperature fluorescence spectra, a part of the mobile LHCII pool was associated with PSI in all light treatments applied in this study.

The plant perceives information on the light environment by means of PSA and the system of specific photoreceptors sensitive to different spectral regions. Any monochromatic light can be regarded as unnatural light conditions to plants. At the same time, a large photon flux density in a narrow spectral range can be identified (misinterpreted) by plants as a high-intensity white light; this view is supported by the data we obtained on the ultrastructure and pigment content. However, the blue and red lights are not equal: red light is perceived in addition 
to PSA by phytochromes only, whereas blue light is absorbed by both phytochromes and blue light receptors (cryptochromes, phototropins). In this respect, blue light exciting a larger set of photoreceptors is functionally more versatile. Indeed, blue light is most effective in stimulating the transcription of photosynthesis-related genes (via cryptochromes and phytochromes) (Liere et al. 2011). We found that the RL-grown plants possessed specific organization of chloroplast membranes (shaggy-formed

\section{References}

Albertsson P.: A quantitative model of the domain structure of the photosynthetic membrane. - Trends Plant Sci. 6: 349-354, 2001.

Allen J.F.: How does protein phosphorylation regulate photosynthesis? - Trends Biochem. Sci. 17: 12-17, 1992.

Allen J.F., Forsberg J.: Molecular recognition in thylakoid structure and function. - Trends Plant Sci. 6: 317-326, 2001.

Anderson J.M., Andersson B.: The dynamic photosynthetic membrane and regulation of solar energy conversion. - Trends Biochem. Sci. 13: 351-355, 1988.

Anderson J.M., Chow W.S., Goodchild D.J.: Thylakoid membrane organisation in sun/shade acclimation. - Aust. J. Plant Physiol. 15: 11-26, 1988.

Arnon D.I.: Copper enzymes in isolated chloroplasts. Polyphenoloxidase in Beta vulgaris. - Plant Physiol. 24: 1-15, 1949.

Beator J., Pötter E., Kloppstech K.: The effect of heat shock on morphogenesis in barley. - Plant Physiol. 100: 1780-1786, 1992.

Belgio E., Ungerer P., Ruban A.V.: Light-harvesting superstructures of green plant chloroplasts lacking photosystems. Plant Cell Environ. 38: 2035-2047, 2015.

Bennett J.: Biosynthesis of the light-harvesting chlorophyll $a / b$ protein. Polypeptide turnover in darkness. - Cur. J. Biochem. 118: 61-70, 1981.

Benson S.L., Maheswaran P., Ware M.A. et al.: An intact light harvesting complex I antenna system is required for complete state transitions in Arabidopsis. - Nat. Plants 176: 1-9, 2015.

Bonaventura C., Myers J.: Fluorescence and oxygen evolution from Chlorella pyrenoidosa. - Biochim. Biophys. Acta 189: 366-383, 1969.

Butler W.L.: Chlorophyll fluorescence: A probe for electron transfer and energy transfer. - In: Trebst A., Avron M. (ed.): Encyclopedia of Plant Physiology. New Series. 5. Pp. 149-167. Springer, Heidelberg 1977.

Chekunova E.M., Savelieva N.V.: [LTS3 gene controls lightindependent chlorophyll biosynthesis in green algae Chlamydomonas reinhardtii.] - Ekol. Gen. 8: 35-44, 2010. [In Russian]

Chow W.S., Melis A., Anderson J.M.: Adjustments of photosystem stoichiometry in chloroplasts improve the quantum efficiency of photosynthesis. - P. Natl. Acad. Sci. USA 87: 7502-7506, 1990.

Chuartzman S.G., Nevo R., Shimoni E. et al.: Thylakoid membrane remodeling during state transitions in Arabidopsis. Plant Cell 20: 1029-1039, 2008.

Deng X.W., Tonkyn J.C., Peter G.F. et al.: Post-transcriptional control of plastid mRNA accumulation during adaptation of chloroplasts to different light quality environments. - Plant Cell 1: 645-654, 1989.

Danielsson R., Albertsson P.-A., Mamedov F. et al.: Quantifi- grana) and light-harvesting complexes (increased energy transfer to PSI, possibly due to spillover promoted by this particular granum structure). These features of RL-grown plants can be related to contradictory information arriving from the photoreceptors: the signal from the phytochromes and photosynthetic apparatus indicates the incidence of light, but the lack of signal from the blue light receptors can be misinterpreted as darkness.

cation of photosystem I and II in different parts of the thylakoid membrane from spinach. - Biochim. Biophys. Acta 1608: 5361, 2004.

Dekker, J.P., Boekema E.J.: Supramolecular organization of thylakoid membrane proteins in green plants. - Biochim. Biophys. Acta 1706: 12-39, 2005.

Dietzel L., Bräutigam K., Steiner S. et al.: Photosystem II supercomplex remodeling serves as an entry mechanism for state transitions in Arabidopsis. - Plant Cell 23: 2964-2977, 2011.

Eskins K., Westhoff P., Beremand P.D.: Light quality and irradiance level interaction in the control of expression of lightharvesting complex of photosystem II. Pigments, pigmentproteins, and mRNA accumulation. - Plant Physiol. 91: 163$169,1989$.

Galka P., Santabarbara S., Khuong T.T.H. et al.: Functional analyses of the plant photosystem I - light-harvesting complex II supercomplex reveal that light-harvesting complex II loosely bound to photosystem II is a very efficient antenna for photosystem I in state II. - Plant Cell 24: 2963-2978, 2012.

Hogewoning S.W., Wientjes E., Douwstra P. et al.: Photosynthetic quantum yield dynamics: from photosystems to leaves. - Plant Cell 24: 1921-1935, 2012.

Iwai M., Takizawa K., Tokutsu R. et al.: Isolation of the elusive supercomplex that drives cyclic electron flow in photosynthesis. - Nature 464: 1210-1213, 2010.

Kouřil R., Wientjes E., Bultema J. B. et al.: High-light vs. lowlight: Effect of light acclimation on photosystem II composition and organization in Arabidopsis thaliana. - BBABioenergetics 1827: 411-419, 2013.

Kouřil R., Zygadlo A., Arteni A.A. et al.: Structural characterization of a complex of photosystem I and lightharvesting complex II of Arabidopsis thaliana. Biochemistry 44: 10935-10940, 2005.

Krause G. H., Weis E.: Chlorophyll fluorescence and photosynthesis: The Basics. - Annu. Rev. Plant Phys. 42: 313-349, 1991.

Ladygin V. G., Semenova G. A.: The involvement of the complexes of photosystems in the organization of chloroplast ultrastructure in pea mutants. - Rus. J. Plant Physl+ 52: 596$605,2005$.

Laisk A., Oja V., Eichelmann H. et al.: Action spectra of photosystems II and I and quantum yield of photosynthesis in leaves in State 1. - Biochim. Biophys. Acta 1837: 315-325, 2014.

Leong T.-Y., Anderson J.M.: Effects of light quality on the composition and function of thylakoid membranes in Atriplex triangularis. - BBA-Bioenergetics 766: 533-541, 1984.

Legocka J., Sobieszczuk-Nowicka E., Wojtyla Ł. et al.: Leadstress induced changes in the content of free, thylakoid- and chromatin-bound polyamines, photosynthetic parameters and ultrastructure in greening barley leaves. - J. Plant Physiol. 186- 
187: 15-24, 2015

Lichtenthaler H.K.: Chlorophyll and carotenoids: pigments of photosynthetic biomembranes. - Methods Enzymol. 148: 350$382,1987$.

Lichtenthaler H.K., Burkart S.: Photosynthesis and high light stress. - Bulg. J. Plant Physiol. 25: 3-16, 1999.

Liere K., Weihe A, Börner T.: The transcription machineries of plant mitochondria and chloroplasts: Composition, function, and regulation. - J. Plant Physiol. 168: 1345-1360, 2011.

Liu X.Y., Guo S.R., Xu Z.G. et al.: Regulation of chloroplast ultrastructure, cross-section anatomy of leaves, and morphology of stomata of cherry tomato by different light irradiations of light-emitting diodes. - HortScience 46: 217-221, 2011.

Liu X., Guo S., Chang T. et al.: Regulation of the growth and photosynthesis of cherry tomato seedlings by different light irradiations of light emitting diodes (LED). - Afr. J. Biotechnol. 11: 6169-6177, 2012.

Melis A.: Light regulation of photosynthetic membrane structure, organization, and function. - J. Cell. Biochem. 24: 271-285, 1984.

Melis A.: Dynamics of photosynthetic membrane composition and function. - BBA-Bioenergetics 1058: 87-106, 1991.

Mullineaux C.: Function and evolution of grana. - Trends Plant Sci. 10: 521-525, 2005.

Murata N.: Control of excitation transfer in photosynthesis. I. Light-induced change of chlorophyll a fluorescence in Porphyridium cruentum. - Biochim. Biophys. Acta 172: 242251, 1969.

Papageorgiou G.C., Govindjee: Photosystem II fluorescence: slow changes - scaling from the past. - J. Photoch. Photobio. B 104: 258-270, 2011.

Pfeiffer S., Krupinska K.: New insights in thylakoid membrane organization. - Plant Cell Physiol. 46: 1443-1451, 2005.

Pribil M., Labs M., Leister D.: Structure and dynamics of thylakoids in land plants. - J. Exp. Bot. 65: 1955-1972, 2014.

Reynolds E.S.: The use of lead citrate at high $\mathrm{pH}$ as an electronopaque stain in electron microscopy. - J. Cell Biol. 17: 208-212, 1963.

Ruban A.V., Johnson M.P.: Visualizing the dynamic structure of the plant photosynthetic membrane. - Nat. Plants 1: 15161, 2015.
Su N., Wu Q., Shen Z. et al.: Effects of light quality on the chloroplastic ultrastructure and photosynthetic characteristics of cucumber seedlings. - Plant Growth Regul. 73: 227-235, 2014

Takahashi H., Iwai M., Takahashi Y. et al.: Identification of the mobile light-harvesting complex II polypeptides for state transition in Chlamydomonas reinhardtii. - P. Natl. Acad. Sci. USA 103: 477-482, 2006.

Tanaka R, Tanaka A.: Chlorophyll $b$ is not just an accessory pigment but a regulator of the photosynthetic antenna. Porphyrins 9: 240-245, 2000.

Tikkanen M., Aro E.-M.: Thylakoid protein phosphorylation in dynamic regulation of photosystem II in higher plants. Biochim. Biophys. Acta 1817: 232-238, 2012.

Tikkanen M., Nurmi M., Suorsa M. et al.: Phosphorylationdependent regulation of excitation energy distribution between the two photosystems in higher plants. - Biochim. Biophys. Acta 1777: 425-432, 2008.

Tikkanen M., Piippo M., Suorsa M. et al.: State transitions revisited - a buffering system for dynamic low light acclimation of Arabidopsis. - Plant Mol. Biol. 62: 779-793, 2006.

Vlasova M.P., Osipova O.P.: [Effect of light intensity on fine structure of the chloroplasts in Vicia faba plants.] - Fiziol. Rast. 20: 742-746, 1973. [In Russian]

Wagner R., Dietzel L., Bräutigam K. et al.: The long-term response to fluctuating light quality is an important and distinct light acclimation mechanism that supports survival of Arabidopsis thaliana under low light conditions. - Planta 228: 573-587, 2008.

Ware M. A., Belgio E., Ruban A.V.: Photoprotective capacity of non-photochemical quenching in plants acclimated to different light intensities. - Photosynth. Res. 126: 261-274, 2015.

Weakley B.S.: A Beginner's Handbook in Biological Electron Microscopy. Pp. 228. Churchill Livingstone, Edinburg and London 1972.

Wientjes E., van Amerongen H., Croce R.: LHCII is an antenna of both photosystems after long-term acclimation. - Biochim. Biophys. Acta 1827: 420-426, 2013.

Yoshioka-Nishimura M.: Close relationships between the PSII repair cycle and thylakoid membrane dynamics. - Plant Cell Physiol. 57: 1115-1122, 2016. 